\title{
Transatlantica
}

Revue d'études américaines. American Studies Journal

$1 \mid 2016$

Modernist Revolutions: American Poetry and the

Paradigm of the New

\section{Conférence d'Emory Douglas, ancien ministre de la Culture des Black Panthers, peintre et illustrateur}

Université Sorbonne Nouvelle - Paris 3, 16 octobre 2015

\section{Alice Morin}

\section{OpenEdition}

Journals

Édition électronique

URL : https://journals.openedition.org/transatlantica/8037

DOI : 10.4000/transatlantica.8037

ISSN : $1765-2766$

Éditeur

Association française d'Etudes Américaines (AFEA)

\section{Référence électronique}

Alice Morin, «Conférence d'Emory Douglas, ancien ministre de la Culture des Black Panthers, peintre et illustrateur », Transatlantica [En ligne], 1 | 2016, mis en ligne le 16 janvier 2017, consulté le 02 février 2023. URL : http://journals.openedition.org/transatlantica/8037 ; DOI : https://doi.org/10.4000/

transatlantica.8037

Ce document a été généré automatiquement le 2 février 2023.

\section{cc) (ㅇ) $\odot$}

Creative Commons - Attribution - Pas d'Utilisation Commerciale - Pas de Modification 4.0 International - CC BY-NC-ND 4.0

https://creativecommons.org/licenses/by-nc-nd/4.0/ 


\section{Conférence d'Emory Douglas, ancien ministre de la Culture des Black Panthers, peintre et illustrateur}

Université Sorbonne Nouvelle - Paris 3, 16 octobre 2015

\section{Alice Morin}

1 Le 16 octobre 2015, le Centre de recherche sur l'Amérique du Nord (CRAN) (équipe CREW, EA 4399) de l'université de la Sorbonne Nouvelle a reçu Emory Douglas, ancien ministre de la Culture des Black Panthers, peintre et illustrateur, pour une conférence spéciale sur son travail et ses activités de militant. Cette intervention, organisée par Pierre Cras, doctorant du CREW, a porté à la fois sur les productions artistiques d'E. Douglas et sur ses activités militantes - approche profondément transdisciplinaire, (re)présentant l'histoire des États-Unis à l'aune de l'avènement d'une nouvelle culture graphique et permettant de croiser l'historiographie sociopolitique avec une approche relevant de la culture matérielle.

2 Après une brève introduction de Pierre Cras, qui prépare une thèse sur les représentations stéréotypées des Noirs dans les films d'animation sous la direction d'Hélène Le Dantec-Lowry, le public a pu revoir ou se familiariser avec l'œuvre d'Emory Douglas pour les Black Panthers, à travers un court documentaire (8 minutes) produit en 2015 par la compagnie indépendante Dress Code, qui retrace son implication dans l'organisation noire et son invention, pour l'occasion, d'un langage graphique réellement singulier.

3 Le contexte du mouvement pour les droits civiques, depuis le début du combat jusqu'à la frustration croissante menant à sa radicalisation et à la création du Black Panther Party (BPP) en 1966, est ainsi bien rappelé. Le film insiste également sur la mise en place d'une presse noire d'abord underground puis assez populaire, mettant en lumière les enjeux de pouvoir économique et politique de l'époque, alors que, comme le rappelle Emory Douglas, " le champ de la publicité était très majoritairement blanc ». Ainsi, on apprend qu'Emory Douglas a rejoint le BPP rapidement après sa création, après déjà quelques années de militantisme l'ayant presque mené à l'incarcération, et 
suite à un passage par la California Youth Authority (institution de redressement dans laquelle il a participé à l'atelier d'imprimerie) et par le San Francisco City College, où il a étudié l'édition de presse. Il commence alors à créer des séries graphiques inspirées de son engagement dans le mouvement étudiant, il est repéré par Bobby Seale et Huey Newton, les créateurs du BPP, et rejoint leur parti émergent, en tant que membre de la communauté et non pas en tant qu'intellectuel : «The idea was to inform, to enlighten and to educate people in the community about the basic issues and to tell the story from our own perspective", déclare-t-il au sujet de l'hebdomadaire qu'il contribue à fonder en 1967 avec Eldridge Cleaver, sur une idée d'Huey Newton. Lancé dans un petit atelier, disposant de moyens très modestes, le journal, sobrement intitulé The Black Panther, atteint pourtant, à son plus fort, un tirage de 400000 exemplaires. On retiendra finalement du film, composé principalement d'interviews d'Emory Douglas, les anecdotes sur la réalisation du journal - comment, par exemple, une contrainte financière (l'impossibilité d'acheter plus d'une couleur, souvent une couleur primaire, variant au fil des numéros, en plus du noir pour l'impression de la publication) se transforma en un signe distinctif de leur production pour la période.

Lors de la conférence proprement dite, Emory Douglas a présenté son travail d'illustrateur sur plusieurs décennies, qui commence avec son engagement auprès des Black Panthers. Il a ainsi évoqué les discussions menées avec les fondateurs du parti afin de faire connaître leurs combats dans des communautés noires, d'abord locales, dans lesquelles les populations avaient souvent un niveau d'instruction peu élevé. La place de l'image était donc primordiale, comme l'était l'invention de symboles forts, renvoyant par là à une politique de visibilité (voire de provocation visuelle) menée d'abord par les premiers militants pour les droits civiques aux États-Unis, puis par leurs héritiers plus radicaux - en réalité, par tous les mouvements contestataires des années soixante et au-delà. D'ailleurs, E. Douglas a tracé un parallèle entre ses recherches artistiques et le poing levé des athlètes Tommie Smith et John Carlos aux Jeux Olympiques de Mexico en 1968, en ce que les photographies de ce geste - concerté à l'avance pour mettre en avant leurs revendications d'égalité - ont eu une forte portée symbolique. Ainsi, Emory Douglas est revenu sur son travail d'élaboration de certaines figures devenues emblématiques du BPP, en particulier la panthère, ou le porc, qui représente ceux qui abusent de leur autorité. Comme il le note, ces images ont transcendé la communauté africaine-américaine et sont reprises par des LatinoAmericains comme le groupe Los Siete de la Raza (leur « Latino brothers ») et par d'autre militants anti-impérialistes, y compris lors de la révolution cubaine. Il souligne également l'existence de "mots-symboles » comme freedom, très récurrent dans les discours, les manifestations et les objets culturels, comme dans la publication The Black Panther ou dans ses autres dessins.

5 Avant de revenir sur cette solidarité, qui a conduit à une internationalisation croissante de sa production, Emory Douglas s'est penché sur le processus d'élaboration de ses illustrations d'alors, destinées à illustrer des articles dans la publication du BPP, et dont il a montré de nombreux exemples. Si l'on y retrouve régulièrement un recours à la provocation, Emory Douglas insiste sur le fait qu'elles sont «based on facts » et que c'est leur interprétation qui est provocante. Il rappelle ainsi le travail d'intériorisation et de " consiousness raising " - alors beaucoup pratiqué et estimé nécessaire par Seale et Newton - qui s'effectue à travers l'émergence d'un style graphique reconnaissable au premier coup d'œil. D'ailleurs, il dit s'être beaucoup inspiré de photographies pour réaliser ses illustrations du journal, probablement pour créer une tension entre le 
réalisme des images et la relecture en question. Il a parfois utilisé des photographies de lynchages dans le Sud pour éveiller les consciences par le choc de ce qu'on ne peut pas (ou plus) ne pas voir, mais aussi des portraits de jeunes scolarisés à Oakland, en Californie, et considérés comme des cas sans espoir, à qui il a redonné une histoire et une voix, atteignant ainsi l'un des buts de l'organisation.

6 À ce sujet, Emory Douglas rappelle à quel point l'action politique et sociale et les images étaient liées, notamment à travers le medium imprimé, et combien la notion de communauté, d'abord locale, puis de plus en plus étendue, jusqu'à atteindre un niveau national, était centrale à la vision des Black Panthers. Il relaie ainsi, par exemple, l'initiative des «free breakfast programs» dans les écoles, qui fut ensuite reprise par l'État de Californie, ce qui tend à montrer l'impact politique réel du parti, au-delà de son seul héritage symbolique. Les initiatives sociopolitiques se multipliant, avec par exemple le «Free Food Program » ou le « Free Clothes Program », elles étaient relayées par l'organe de presse, qui sollicitait des dons afin de les mener à bien, de redresser et d'éduquer des communautés, tout en renforçant et en amplifiant ainsi les réseaux locaux existants. Cependant, la dimension de provocation, mais surtout de dénonciation, reste une composante importante de l'esprit du journal. Ainsi, lorsque l'État californien investit dans des hélicoptères pour prévenir la criminalité dans certains quartiers sensibles, le Black Panther pointe du doigt la mauvaise gestion d'un argent qui aurait été mieux employé à l'éducation des jeunes dans les quartiers défavorisés - question toujours très actuelle.

7 De même, des événements nationaux contre lesquels le BPP s'élève publiquement sont caricaturés par celui qui devient bientôt leur «ministre de la Culture et de l'Information " (titre qui rappelle les liens entre information et propagande en temps de guerre, «chaude » ou froide) : les jeunes Noirs mis en prison de manière quasiment systématique sont dépeints en "prisonniers politiques », tandis que le «face trial» de Bobby Seale est mis en scène dans les pages du Black Panther, et que les violences policières y sont régulièrement dénoncées et mises en regard avec des boîtes d'allumettes ornées de visages colorés, symboles d'une résistance noire prête à s'enflammer. Le journal dénonce aussi la collusion entre le système raciste et le système capitaliste, mettant en parallèle l'exploitation des Noirs des villes et des ouvriers agricoles latinos qui tentent alors de se syndiquer, ce que la publication soutient, tandis que le système pénitentiaire est lui aussi dénoncé pour sa volonté de prospérer aux dépens des Africains-Américains.

C'est cet esprit que Douglas et d'autres membres du parti ont souhaité s'ouvrir à d'autres combats «frères". Ainsi, une forte solidarité avec certains groupes d'Amérique latine (notamment les mouvements étudiants mexicains à partir de 1968) donnera naissance à des affiches que Douglas destinait à des réunions et à des événements internationaux, mais aussi à des œuvres hybrides. Un poster de Lazaro Abreu pour l'OSPAAAL (Organization of Solidarity of the People of Africa, Asia and Latin America) à Cuba illustre ce phénomène : représentant des guérilleros aux origines variées et surtitré "Solidarity with the African American People» (1968), il reprend deux illustrations de Douglas en modifiant les couleurs originales de ces deux dessins et en recentrant le propos autour de questionnements plus étendus - permettant par la même occasion au coup de crayon de l'auteur original de se faire connaître internationalement. 
Une telle expansion correspond d'ailleurs à la chute du mouvement des Black Panthers, sapé par la section CoInTelPro du FBI qui (comme le rappelle le film documentaire) tente notamment de saper le journal, en retournant les imprimeurs et fabricants contre les créateurs, profitant de leur jeunesse et de leur manque d'expérience. Mais alors que l'organisation se défait, son héritage commence à grandir et ses symboles prennent une vie propre. L'œuvre postérieure d'Emory Douglas se développe à partir de là, avec, d'une part, une série de portraits des grands leaders noirs héroïsés (Malcolm X the Warrior, Martin Luther King the Dr. etc.) stylisés, très colorés, comme en mouvement ; et avec, d'autre part, une série de portraits de Noirs pauvres, travailleurs agricoles ou ouvriers dans l'industrie, à qui il continue ainsi de prêter voix. Après plusieurs décennies, ce travail de dissémination d'un héritage et d'une mémoire donnera lieu à sa production de "celebration posters", réutilisant ses orignaux de l'époque comme base de collages, mêlant superpositions et télescopage de symboles. Un autre aspect de la continuité entre ses activités au sein du BPP et son travail plus récent est son implication militante récurrente, au fil des années, dans des pays tels que l'Afrique du Sud ou la Nouvelle-Zélande, entre autres, et son constant exercice de la satire politique, comme en témoignent la reprise d'un portait d'Obama avec pour légende «Nobel fraud » ou encore la dénonciation de la corruption capitaliste, couplée avec l'action sur le terrain. À ce titre, Emory Douglas rappelle son travail avec des enfants à Manchester, au Royaume-Uni, pour les aider à augmenter leur estime d'eux-mêmes.

Il est intéressant de noter, pour conclure, que ce travail, s'il est toujours en cours et en constante évolution (la critique et les réflexions offertes lors de la conférence s'inscrivant bien dans la perspective d'une relecture contemporaine), intéresse de plus en plus les institutions, comme on l'a vu avec le travail de mémoire. Il fait l'objet d'une patrimonialisation croissante, avec l'organisation d'expositions personnelles dans des lieux culturels reconnus (au New Museum de New York, au MoCA ou à la galerie Ze Dos Bois de Lisbonne), où sont installées des fresques commémoratives rassemblant ses œuvres les plus célèbres, mais aussi des réinterprétations modernes plus ou moins populaires de ces dernières (revisitées, par exemple, sous forme de broderies). Emory Douglas lui-même semble approuver un tel usage de sa production qu'il faut, considère$\mathrm{t}$-il, reprendre, retravailler sans cesse et adapter aux causes actuelles.

\section{INDEX}

Thèmes : Actualité de la recherche

\section{AUTEUR}

\section{ALICE MORIN}

Université Sorbonne Nouvelle - Paris 3 